\title{
A v-INTEGRAL REPRESENTATION FOR LINEAR OPERATORS ON A SPACE OF CONTINUOUS VECTOR-VALUED FUNCTIONS
}

\author{
J. R. EDWARDS AND S. G. WAYMENT
}

ABstract. In this note an analytic representation is given for continuous linear operators from $C(X)$ into a linear normed space $Y$ where $C(X)$ is the space of continuous functions on $[0,1]$ with values in a linear normed space $X$.

1. Introduction. With a very elaborate and difficult construction D. H. Tucker [3] obtains a Stieltjes-type integral representation for continuous linear operators from $C(X)$ in to a linear normed space $Y$, where $C(X)$ is the space of continuous functions on $[0,1]$ with values in a linear normed space $X$. In this note we give a representation theorem in terms of the $v$-integral (introduced in [1]) and the argument is straightforward.

2. The representation theorem. Suppose $K$ is a set function defined on half open intervals $(a, b] \subset[0,1]$ with values in $B[X, Y]$, the space of bounded linear operators from $X$ into $Y$. If there is a constant $M$ such that for any disjoint collection of such intervals $\left\{I_{i}\right\}$ and any corresponding collection $\left\{x_{i}\right\} \subset X,\left\|\sum\left\{K\left(I_{i}\right)\right\}\left(x_{i}\right)\right\| \leqq M$ $\cdot \max _{j}\left\|\sum_{i=1}^{j} x_{i}\right\|$, then $K$ is said to be convex-Gowurin, and the smallest such constant $M$ shall be denoted by $W K$. Let $T$ be a continuous linear operator from $C(X)$ into $Y$. Since

$$
T(f)=T\left(f-\chi_{[0,1]} f(0)\right)+T\left(\chi_{[0,1]} f(0)\right)
$$

we shall consider $T$ restricted to $C_{\theta}(X)$, the subset of functions $f \in C(X)$ satisfying $f(0)=\theta$.

THEOREM 1. If $K$ is a set function with values in $B[X, Y]$ which is convex-Gowurin and convex with respect to length $[1]$, then $T(f)=v \int K d f$ is a bounded linear operator from $C_{\theta}(X)$ to $\bar{Y}$, the completion of $Y$, and $\|T\|=W K$.

Proof. Suppose $\sigma$ and $\sigma^{\prime}$ are partitions of $[0,1]$ and suppose $f \in C_{\theta}(X)$. Then,

Received by the editors December 8, 1969.

AMS 1969 subject classifications. Primary 2825, 4725; Secondary 4625.

Key words and phrases. Continuous linear operator, vector-valued function, representation theorem, convex-Gowurin, $v$-integral, polygonal function. 


$$
\begin{aligned}
& \left\|\sum_{\sigma}\left\{K\left(\left(t_{i}, t_{i+1}\right]\right)\right\}\left(\Delta_{i} f\right)-\sum_{\sigma^{\prime}}\left\{K\left(\left(t_{j}, t_{j+1}\right]\right)\right\}\left(\Delta_{i} f\right)\right\| \\
& \leqq \| \sum_{\sigma^{*} \sigma^{\prime}}\left\{K\left(\left(t_{\nu}, l_{\nu+1}\right)\right\}\left(\Delta_{\nu}\left(p f_{\sigma}-p f_{\sigma^{\prime}}\right)\right) \|\right. \\
& \leqq W K \max _{\mu}\left\|\sum_{\nu=1}^{\mu} \Delta_{\nu}\left(p f_{\sigma}-p f_{\sigma^{\prime}}\right)\right\|,
\end{aligned}
$$

where $p f_{\sigma}$ denotes the polygonal function determined by $f$ and $\sigma$. As the mesh-fineness of $\sigma$ and $\sigma^{\prime}$ tend to zero, the above tends to zero. Hence, $T(f)=v \int K d f$ exists. That $T$ is a bounded operator with $\|T\|$ $\leqq W K$ follows by a similar argument and is omitted.

Let $\Psi_{I}$ denote the fundamental function of the interval $I[1]$. That is, if $I=(a, b]$, then

$$
\begin{aligned}
\Psi_{I} & =0 & & \text { if } t \leqq a, \\
& =(t-a) /(b-a) & & \text { if } a \leqq t \leqq b, \\
& =1 & & \text { if } t \geqq b .
\end{aligned}
$$

Then $\|T\| \geqq W K$, since for $\left\{I_{i}\right\}$ disjoint in $[0,1]$ and $\left\{x_{i}\right\} \subset X$,

$$
\begin{aligned}
\left\|\sum\left\{K\left(I_{i}\right)\right\}\left(x_{i}\right)\right\| & =\left\|v \int K\left(\sum \Psi_{I_{i}} x_{i}\right)\right\|=\left\|T\left(\sum \Psi_{I_{i}} x_{i}\right)\right\| \\
& \leqq\|T\| \cdot\left\|\sum \Psi_{I_{i}} \cdot x_{i}\right\|_{\infty} \leqq\|T\| \max _{j}\left\|\sum_{i=1}^{j} x_{i}\right\| .
\end{aligned}
$$

From the two preceding inequalities we conclude $\|T\|=W K$.

THEOREM 2. Suppose $T$ is a bounded linear operator from $C_{\theta}(X)$ into $Y$. Then there is a unique set function $K$ with values in $B[X, Y]$ which is convex-Gowurin and convex with respect to length such that $T(f)=v \int K d f$ for each $f \in C_{\theta}(X)$. Furthermore, $\|T\|=W K$.

Proof. Define $J$ from the continuous real valued functions on $[0,1]$ into $B[X, Y]$ by $[J(f)](x)=T(f \cdot x)$. It follows as in [2] that $\|\Im\| \leqq\|T\|$. Define the set function $K$ on the intervals in $[0,1]$ by $K(I)=\Im\left(\Psi_{I}\right)$. That $K$ is convex with respect to length follows from the linearity of $J$ and from the manner in which fundamental functions combine. Observe that, for disjoint half open intervals $\left\{I_{i}\right\}$ in $[0,1]$ and $\left\{x_{i}\right\} \in X$,

$$
\begin{aligned}
\left\|\sum\left\{K\left(I_{i}\right)\right\}\left(x_{i}\right)\right\| & =\left\|\sum\left\{J\left(\Psi_{I_{i}}\right)\right\}\left(x_{i}\right)\right\|=\left\|T\left(\sum \Psi_{I_{i}} \cdot x_{i}\right)\right\| \\
& \leqq\|T\| \cdot\left\|\sum \Psi_{I_{i}} x_{i}\right\|_{\infty}=\|T\| \max _{j}\left\|\sum_{i=1}^{j} x_{i}\right\|,
\end{aligned}
$$


hence, $K$ is convex-Gowurin. Suppose $f \in C_{\theta}(X)$, then

$$
\begin{aligned}
T(f) & =\lim _{\sigma} T\left(p f_{0}\right)=\lim _{\sigma} T\left(\sum_{\sigma} \Psi_{I_{i}} \Delta_{i} f\right)=\lim _{\sigma} \sum_{\sigma}\left\{K\left(I_{i}\right)\right\}\left(\Delta_{i} f\right) \\
& =v \int K d f .
\end{aligned}
$$

The last equality follows from Theorem 1 as does the fact that $\|T\|$ $=W K$. Since $K$ determines $T$ uniquely on polygonal functions, which are dense in $C(X), K$ is unique.

Hence, in the case $Y$ is complete, the representation given here is a characterization of the linear tranformations. Such a characterization is not immediate from [2] and [3].

\section{REFERENCES}

1. J. R. Edwards and S. G. Wayment, Representation for transformations continuous in the BV norm, Trans. Amer. Math. Soc. 154 (1971), 251-265.

2. D. H. Tucker, A note on the Riesz representation theorem, Proc. Amer. Math. Soc. 14 (1963), 354-358. MR 26 \#2865.

3. - A representation theorem for a continuous linear transformation on a space of continuous functions, Proc. Amer. Math. Soc. 16 (1965), 946-953. MR 33 \#7865.

Utah State University, Logan, Utah 84321 